\title{
Article
}

\section{The influence of barefoot and barefoot inspired footwear on the kinetics and kinematics of running in comparison to conventional running shoes.}

Sinclair, Jonathan Kenneth, Greenhalgh, Andrew, Brooks, Darrell, Edmundson, Christopher James and Hobbs, Sarah Jane

Available at http://clok.uclan.ac.uk/4346/

Sinclair, Jonathan Kenneth ORCID: 0000-0002-2231-3732, Greenhalgh, Andrew, Brooks, Darrell ORCID: 0000-0002-4094-5266, Edmundson, Christopher James ORCID: 0000-0003-2275-7584 and Hobbs, Sarah Jane ORCID: 0000-0002-1552-8647 (2012) The influence of barefoot and barefoot inspired footwear on the kinetics and kinematics of running in comparison to conventional running shoes. Footwear Science, 5 (1).

It is advisable to refer to the publisher's version if you intend to cite from the work. http://dx.doi.org/10.1080/19424280.2012.693543

For more information about UCLan's research in this area go to http://www.uclan.ac.uk/researchgroups/ and search for <name of research Group>.

For information about Research generally at UCLan please go to http://www.uclan.ac.uk/research/

All outputs in CLoK are protected by Intellectual Property Rights law, including Copyright law. Copyright, IPR and Moral Rights for the works on this site are retained by the individual authors and/or other copyright owners. Terms and conditions for use of this material are defined in the policies page. 


\section{$1 \quad \underline{\text { Abstract }}$}

2 Barefoot running has experienced a resurgence in footwear biomechanics literature, based on the supposition that it serves to reduce the occurrence of overuse injuries in comparison to conventional shoe models. This consensus has lead footwear manufacturers to develop shoes which aim to mimic the mechanics of barefoot locomotion.

This study compared the impact kinetics and 3-D joint angular kinematics observed whilst running: barefoot, in conventional cushioned running shoes and in shoes designed to integrate the perceived benefits of barefoot locomotion. The aim of the current investigation was therefore to determine whether differences in impact kinetics exist between the footwear conditions and whether shoes which aim to simulate barefoot movement patterns can closely mimic the 3-D kinematics of barefoot running.

Twelve participants ran at $4.0 \mathrm{~m} \cdot \mathrm{s}^{-1} \pm 5 \%$ in each footwear condition. Angular joint kinematics from the hip, knee and ankle in the sagittal, coronal and transverse planes were measured using an eight camera motion analysis system. In addition simultaneous tibial acceleration and ground reaction forces were obtained. Impact parameters and joint kinematics were subsequently compared using repeated measures ANOVAs.

The kinematic analysis indicates that in comparison to the conventional and barefoot inspired shoes that running barefoot was associated significantly greater plantar-flexion at footstrike and range of motion to peak dorsiflexion. Furthermore, the kinetic analysis revealed that compared to the conventional footwear impact parameters were significantly greater in the barefoot condition.

Therefore this study suggests that barefoot running is associated with impact kinetics linked to an increased risk of overuse injury, when compared to conventional shod running. Furthermore, the mechanics of the shoes which aim to simulate barefoot movement patterns do not appear to closely mimic the kinematics of barefoot locomotion. 


\section{Introduction}

In recent years the concept of barefoot running has been the subject of much attention in footwear biomechanics literature. Furthermore, a number of well known athletes have competed barefoot, most notably Zola Budd-Pieterse and the Abebe Bikila who both held world records for the $5000 \mathrm{~m}$ and marathon events respectively. This demonstrates that barefoot running does not appear to prevent athletes from competing at the highest levels (Warburton 2001). Barefoot locomotion presents a paradox in footwear literature (Robbins and Hanna 1987); and has been used for many years both by coaches and athletes (Nigg 2009) based around the supposition that running shoes are associated with an increased incidence of running injuries (Lieberman et al., 2010, Robbins and Hanna 1987; Warburton 2001).

Based on such research and taking into account the barefoot movement's recent rise in popularity, shoes have been designed in an attempt to transfer the perceived advantages of barefoot movement into a shod condition (Nigg 2009). Yet, given the popularity of barefoot running, surprisingly few investigations have specifically examined the both the impact kinetics and 3-D kinematics of the lower extremities of running barefoot and in barefoot inspired footwear in comparison to shod. Furthermore, there is a paucity of research reporting the prospective epidemiological investigations into the aetiology of injury in runners and how footwear may affect the frequency of injury. This study provides a comparison of the kinetics and 3-D kinematics of running: barefoot, in conventional running shoes and in barefoot inspired footwear, in order to highlight the differences among conditions.

The aim of the current investigation was therefore to determine 1: whether differences in impact kinetics during running exist between the footwear conditions and 2: whether shoes 
which aim to simulate barefoot movement patterns can closely mimic the 3-D kinematics of barefoot running.

\section{Methods}

\section{Participants}

The procedure utilized for this investigation was approved by the University of Central Lancashire, School of Psychology, ethical committee. Twelve experienced male runners completing at least $30 \mathrm{~km}$ per week, volunteered to take part in this study. All were injury free at the time of data collection and provided written informed consent. The mean characteristics of the participants were; age $24.34 \pm 1.10$ years, height $178.10 \pm 5.20 \mathrm{~cm}$ and body mass $76.79 \pm 8.96 \mathrm{~kg}$. A statistical power analysis was conducted using $\mathrm{G}^{*}$ Power Software using a moderate effect size (Erdfelder et al., 1996), to reduce the likelihood of a type II error and determine the minimum number participants needed for this investigation. It was found that the sample size was sufficient to provide more than $80 \%$ statistical power.

\section{Procedure}

Participants ran at $4.0 \mathrm{~m} . \mathrm{s}^{-1}$ over a force plate (Kistler, Kistler Instruments Ltd., Alton, Hampshire) embedded in the floor (Altrosports 6mm, Altro Ltd,) of a $22 \mathrm{~m}$ biomechanics laboratory. Running velocity was quantified using Newtest 300 infrared timing gates (Newtest, Oy Koulukatu, Finland), a maximum deviation of $\pm 5 \%$ from the set velocity was allowed. Stance time was defined as the time over which $20 \mathrm{~N}$ or greater of vertical force was applied to the force platform (Sinclair et al., 2011). A successful trial was defined as one within the specified velocity range, where all tracking clusters were in view of the cameras, the foot made full contact with the force plate and no evidence of gait modifications due to the experimental conditions. Runners completed a minimum of six successful trials in each 
footwear condition. Participants were non-habitual barefoot runners and were thus given time to accommodate to the barefoot and barefoot inspired footwear prior to the commencement of data collection. This involved 5 minutes of running through the testing area without concern for striking the force platform.

Kinematics and tibial acceleration data were also synchronously collected. Kinematic data was captured at $250 \mathrm{~Hz}$ via an eight camera motion analysis system (Qualisys Medical AB, Goteburg, Sweden). Calibration of the system was performed before each data collection session. Only calibrations which produced average residuals of less than $0.85 \mathrm{~mm}$ for each camera for a $750.5 \mathrm{~mm}$ wand length and points above 3000 in all cameras were accepted prior to data collection.

The marker set used for the study was based on the calibrated anatomical systems technique (CAST) (Cappozo et al., (1995). In order to define the right foot, shank and thigh retroreflective markers were attached unilaterally to the 1 st and 5th metatarsal heads, medial and lateral maleoli, medial and lateral epicondyle of the femur and greater trochanter. To define the pelvis additional retro-reflective markers were placed on the anterior (ASIS) and posterior (PSIS) superior iliac spines. Rigid tracking clusters were positioned on the shank and thigh. Each rigid cluster comprised four $19 \mathrm{~mm}$ diameter spherical reflective markers mounted to a thin sheath of lightweight carbon fibre with length to width ratios in accordance with Cappozzo et al., (1997). A static trial was conducted with the participant in the anatomical position in order for the positions of the anatomical markers to be referenced in relation to the tracking clusters, following which they were removed.

A tri-axial (Biometrics ACL 300, Gwent United Kingdom) accelerometer sampling at $1000 \mathrm{~Hz}$ was utilized to measure axial accelerations at the tibia. The device was mounted on a piece of 
lightweight carbon-fibre material using the protocol outlined by Sinclair et al., (2010). The combined weight of the accelerometer and mounting instrument was 9g. The voltage sensitivity of the signal was set to $100 \mathrm{mV} / \mathrm{g}$, allowing adequate sensitivity with a measurement range of $\pm 100 \mathrm{~g}$. The device was attached securely to the distal anterio-medial aspect of the tibia in alignment with its longitudinal axis $8 \mathrm{~cm}$ above the medial maleolus. This location was selected to attenuate the influence ankle rotation can have on the acceleration magnitude (Lafortune \& Hennig, 1991). Strong non-stretch adhesive tape was placed over the device and leg to avoid overestimating the acceleration due to tissue artefact.

\section{Data Processing}

Trials were processed in Qualisys Track Manager in order to identify anatomical and tracking markers then exported as C3D files. Kinematic parameters were quantified using Visual 3-D (C-Motion Inc, Gaithersburg, USA) after marker data were smoothed using a low-pass Butterworth $4^{\text {th }}$ order zero-lag filter at a cut off frequency of $10 \mathrm{~Hz}$. This frequency was selected as being the frequency at which $95 \%$ of the signal power was below. 3-D kinematics of the hip knee and ankle joints were calculated using an XYZ cardan sequence of rotations (where $\mathrm{X}$ is flexion-extension; $\mathrm{Y}$ is ab-adduction and is $\mathrm{Z}$ is internal-external rotation). All data were normalized to $100 \%$ of the stance phase then processed gait trials were averaged. 3-D kinematic measures from the hip, knee and ankle which were extracted for statistical analysis were 1) angle at footstrike, 2) angle at toe-off, 3) range of motion during stance, 4) peak angle during stance and 5) relative range of motion from footstrike to peak angle.

The acceleration signal was filtered using a $60 \mathrm{~Hz}$ Butterworth zero-lag 4th order low pass filter in accordance with the Lafortune and Hennig, (1992) recommendations to prevent any resonance effects on the acceleration signal. Peak positive axial tibial acceleration was 
defined as the highest positive acceleration peak measured during the stance phase. To analyze data in the frequency domain, a fast fourier transformation function was performed and median power frequency content of the acceleration signals were calculated.

Forces were reported in bodyweights (BWs) to allow normalisation of the data among participants. From the force plate data, peak braking and propulsive forces, stance time, average loading rate, instantaneous loading rate, peak impact force and time to peak impact were calculated. Average loading rate was calculated by dividing the impact peak magnitude by the time to the impact peak. Instantaneous loading rate was quantified as the maximum increase in vertical force between frequency intervals.

\section{Shoes}

The shoes utilized during this study consisted of a Saucony Pro Grid Guide 2 and a Nike Free 3.0. The shoes were the same for all runners; they differed in size only (sizes 6, 7 and 9 in men's shoe UK sizes).

\section{$\underline{\text { Statistical Analysis }}$}

Descriptive statistics including means and standard deviations of 3-D kinematic, impact shock and impact force parameters were calculated for each footwear condition. Differences between the parameters were examined using repeated measures ANOVA's with significance accepted at the $\mathrm{p} \leq 0.05$ level. Appropriate post-hoc analyses were conducted using a Bonferroni correction to control for type I error. Effect sizes were calculated using a $\mu^{2}$. If the sphericity assumption was violated then the degrees of freedom were adjusted using the Greenhouse Geisser correction. The Shapiro-Wilk statistic for each footwear condition 
confirmed that all data were normally distributed. All statistical procedures were conducted using SPSS 19.0 (SPSS Inc, Chicago, USA).

Results

Figure 1 presents the mean 3-D angular kinematics of the hip, knee and ankle joints during the stance phase. Tables 1-4 present the kinetic and 3-D kinematic parameters observed as a function of footwear.

Kinetic Results

@@@TABLE1 NEAR HERE@@@

The results indicate that a significant main effect was observed for the instantaneous loading rate $\mathrm{F}_{(1.08,11.88),}=20.05, \mathrm{p} \leq 0.01, \mu^{2}=0.65$. Post-hoc analyses revealed that the instantaneous loading rate was significantly higher in the barefoot condition in comparison to the footwear designed to simulate barefoot locomotion $(\mathrm{p}=0.011)$ and conventional shoe $(\mathrm{p}=0.001)$ conditions). Furthermore the post-hoc analysis also showed that the footwear designed to simulate barefoot locomotion was associated with a significantly $(\mathrm{p}=0.001)$ higher instantaneous loading rate than the conventional shoe condition. In addition a significant main effect was also observed for the average loading rate $\mathrm{F}_{(1.08,11.84)}=9.19, \mathrm{p} \leq 0.01, \mu^{2}=0.46$. Post-hoc analyses revealed that the average loading rate was significantly lower in the conventional shoe condition in comparison to the shoes designed to simulate barefoot running $(p=0.004)$ and barefoot conditions $(\mathrm{p}=0.02)$ which did not differ significantly $(\mathrm{p}=0.084)$ from one another. A significant main effect was observed for the time to impact peak $\mathrm{F}_{(1.23,13.58)}=7.94, \mathrm{p} \leq 0.01, \mu^{2}=0.41$. Post- 
hoc analyses revealed that the time to impact peak was significantly greater in the conventional shoe condition in comparison to the shoes designed to simulate barefoot running $(\mathrm{p}=0.006)$ and barefoot $(\mathrm{p}=0.042)$ conditions which did not differ significantly $(\mathrm{p}=0.504)$ from one another. Finally, a significant main effect $F_{(1.21,13.35)}=15.81, p \leq 0.01, \mu^{2}=0.59$ was found for the magnitude of peak axial impact shock. Post-hoc analysis revealed that peak impact shock was significantly greater in the barefoot $\mathrm{p}=0.021$ and shoes designed to simulate barefoot running $\mathrm{p}=0.01$ conditions in comparison to the conventional shoe condition. The spectral analysis of the acceleration signal revealed that a significant main effect $\mathrm{F}_{(1.29,14.14)} 14.09, \mathrm{p} \leq 0.01, \mu^{2}=0.56$ existed for the median frequency content. Post-hoc analysis revealed that the conventional shoe condition was associated with a significantly lower frequency content than the barefoot $\mathrm{p}=0.001$ and shoes designed to simulate barefoot conditions $p=0.0001$. No significant differences were observed between the barefoot and shoes designed to simulate barefoot conditions $\mathrm{p}=0.35$. Finally, a significant main effect $\mathrm{F}_{(2,22)}=8.10, \mathrm{p} \leq 0.01, \mu^{2}=0.42$ was found for the stance time duration. Post-hoc analysis revealed that stance times were significantly shorter in the barefoot $\mathrm{p}=0.003$ and the shoes designed to simulate barefoot $\mathrm{p}=0.008$ conditions in comparison to the conventional shoe condition. No significant differences $\mathrm{p}=0.512$ were found between the barefoot and shoes designed to simulate barefoot running.

Kinematic results

@@@FIGURE1 NEAR HERE@@@

\section{Hip}


194 A significant main effect $\mathrm{F}_{(1.25,13.73)}=5.24, \mathrm{p} \leq 0.05, \mu^{2}=0.32$ was found for peak flexion. 195 Post-hoc analysis revealed that peak flexion was significantly $\mathrm{p}=0.039$ greater in the 196 conventional shoe condition, in comparison to the barefoot condition.

197

$198 \quad$ Knee

199

@@@TABLE3 NEAR HERE@@@

No significant $(\mathrm{p} \leq 0.05)$ differences were in knee joint kinematics were found among footwear conditions.

Ankle

@@ @TABLE4 NEAR HERE@@@

A significant main effect $\mathrm{F}_{(2,22)}=7.91, \mathrm{p} \leq 0.01, \mu^{2}=0.42$ was observed for the magnitude of plantarflexion at foot strike. Post-hoc analysis revealed that in the barefoot condition the ankle was significantly more plantar flexed than in both the conventional $\mathrm{p}=0.01$ and the shoes designed to simulate barefoot running $\mathrm{p}=0.015$. A significant main effect $\mathrm{F}_{(1.06,11.66)}=8.23$, hoc analyses revealed that this motion was significantly greater in the barefoot condition in comparison to the barefoot inspired footwear $\mathrm{p}=0.011$ and conventional shoe $\mathrm{p}=0.013$ conditions.

214 The results indicate that a significant main effect $\mathrm{F}_{(2,22)}=7.23, \mathrm{p} \leq 0.01$, Eta $^{2}=0.40$ exists for the magnitude of peak axial rotation. Post-hoc analysis revealed that the barefoot condition 
was significantly $\mathrm{p}=0.001$ more externally rotated in comparison to the shoes designed to simulate barefoot running. The results indicate that a significant main effect $F_{(2,22)}=6.09$, $\mathrm{p} \leq 0.01, \mu^{2}=0.36$ exists for the magnitude of axial rotation at toe-off. Post-hoc analysis revealed that external rotation was significantly $\mathrm{p}=0.001$ greater in the barefoot condition in comparison to the shoes designed to simulate barefoot running.

\section{Discussion}

This study represents is the first to examine synchronously examine alterations in 3-D kinematics, force and axial impact shock associated with running barefoot, in conventional footwear and in footwear designed to simulate barefoot running.

The results from the kinetic analysis indicate that the conventional shoes were associated with lower impact parameters than running barefoot. This finding corresponds with the results of previous investigations (Dickinson et al., 1985, De Koning and Nigg 1993, De Clercq et al., 1994 and De Wit et al., 2000) who reported significantly greater impact parameters when running barefoot. This however opposes the findings of Squadrone and Gallozzi (2009) and Lieberman et al., (2010) who observed that those running barefoot were associated with smaller collision forces than shod. Moreover, that instantaneous loading rate was found to be significantly greater in the barefoot condition in comparison to the barefoot inspired shoes opposes the findings of Squadrone and Gallozzi (2009) who reported that impact forces did not differ significantly between barefoot and barefoot inspired footwear. These observations may relate to the differences in barefoot running experience between studies. Squadrone and Gallozzi (2009) and Lieberman et al., (2010) utilized habitual barefoot runner which is in contrast to the non-habitual barefoot runners examined in the current investigation. Therefore the kinetic observations in barefoot analyses may relate to the experience of the participants in 
barefoot locomotion, this is an interesting notion and future research may wish to replicate the current investigation using habitually barefoot runners.

The results also indicate that stance times were significantly shorter whilst running barefoot and in barefoot inspired footwear in comparison to the conventional running shoe condition. This also corresponds with previous investigations with respect to shorter stance times being associated with barefoot running (De Wit et al., 2000, Warburton 2001). Furthermore it would also appear to confirm that the barefoot condition was associated with a greater step frequency/reduced step lengths, as De Wit et al., (2000) found stance times to be strongly correlated with step length. With respect to the hip joint complex, in the sagittal plane a significant increase in peak flexion during the early stance phase was found in the conventional shoe condition in comparison to the barefoot condition. It is surmised that this finding is attributable to the mechanical alterations that runners make when running barefoot (as described above). Runners traditionally take longer steps when running in traditional footwear, so their centre of mass moves through a greater horizontal displacement during each step. As such, during early stance the hip must flex to a greater extent in order to reduce the horizontal distance from the stance leg to the centre of mass to maintain balance during the early stance phase.

The results indicate that the ankle was significantly more plantar flexed at initial contact in the barefoot condition in comparison to the conventional shoe and barefoot inspired footwear, suggesting a mid or forefoot strike pattern. This concurs with the findings of (De Wit et al., 2000, Hartveld and Chockalingam 2001 and Griffin et al., 2007) findings. Barefoot running or running in shoes with less midsole cushioning is proposed to facilitate increases in plantar 
discomfort which are sensed and moderated (Robbins and Gouw, 1991). Footwear with greater cushioning i.e. the conventional and barefoot inspired footwear conditions provoke a reduction in shock-moderating behaviour as evidenced by the increased dorsiflexion angle at footstrike (Robbins and Hanna, 1987; Robbins et al., 1989; Robbins and Gouw, 1991). This may lend support to the supposition that the body adapts to a lack of cushioning via kinematic measures. However, it appears that these measures do not offer the same shock attenuating properties as do cushioned midsoles found in conventional footwear.

The increase in plantarflexion at footstrike associated with barefoot running is considered to be the primary mechanism by which runners adjust to this condition (De Wit et al., 2000, Warburton 2001 and Griffin et al., 2007). Thus, it appears that the barefoot inspired footwear do not closely mimic the kinematics of barefoot running with respect to the ankle joint complex. It is proposed that this finding is attributable to the perceptual effects of increased cushioning in the barefoot inspired footwear which were found to have increased shock attenuating properties. This finding opposes the observations of Squadrone and Gallozzi (2009) who found that barefoot inspired footwear where effective in imitating barefoot conditions. However, Squadrone and Gallozzi (2009) utilized the vibram five-fingers which are characterized by their minimalist features in contrast to the Nike Free footwear utilized in the current investigation which aims to simulate barefoot locomotion through a flexible outsole construction. BAREFOOT SHOES ARE NOT ALL THE SAME THEREFORE Future research is necessary to examine the efficacy of the various conceptual shoe models which aim to replicate barefoot locomotion. 
Interestingly, no significant differences were found between the three footwear conditions, in terms of the peak eversion magnitude during stance. This is appears to oppose the findings of Warburton (2001), Shorten (2000), Edington et al,. (1990), Stacoff et al., (1991) and Smith et al., (1986) who reported that ankle eversion is greater during shod running. Greater ankle eversion is reputed to be due to a reduction in stability caused by the cushioned midsole (Shorten 2000). However like most modern footwear, both the conventional and barefoot inspired footwear encompass features stiffer cushioning, stiff heel counters, insole boards, medially posted midsoles, varus wedges designed to control excessive ankle eversion. Therefore, whilst it appears logical that cushioning will lead to increased ankle eversion the results of this investigation suggest that a combination of cushioning and features designed to control pronation can be effective.

There is a paucity of research directly comparing injury rates in shod and barefoot running. However, the findings of this study in conjunction with epidemiological analyses suggest that running in conventional footwear may lower the incidence of impact related overuse injuries as increases in impact parameters have been linked to the aetiology of a number overuse pathologies (Hardin et al., 2003; Misevich and Cavanagh 1984). Furthermore, the results of the kinetic analysis suggest that the barefoot inspired footwear offer shock attenuating properties that are superior to barefoot conditions, but inferior to the conventiofnal running shoe. Thus it appears based on the findings from the impact kinetic analysis that the footwear designed to mimic barefoot running places runners at greater risk of musculoskeletal injuries compared to the conventional footwear but lesser risk in comparison to barefoot running at comparable velocities. 
That this investigation quantified barefoot locomotion with skin mounted markers and shod motion using shoe mounted markers may serve as a limitation of the current investigation. There is almost certain be movement of the foot within the shoe, thus it is questionable as to whether anatomical markers located on the shoe provide comparable results to those placed on the foot itself Stacoff et al., (1992). However, given that cutting holes in the shoes in order to attach markers to skin would likely cause further problems by compromising the structural integrity of the upper, it was determined that the current technique was the most appropriate.

In conclusion although previous studies have compared barefoot and shod running, the current knowledge with respect to the degree in which these modalities differ is limited. The present study adds to the current knowledge of barefoot running by providing a comprehensive kinetic and 3-D kinematic evaluation. Furthermore, this study is the first to contrast synchronous 3-D kinematic and kinetic variables against barefoot inspired footwear. Given that significant differences were observed between running barefoot and in barefoot inspired footwear, it was determined that they do not closely mimic the mechanics of barefoot running. Future research will serve to determine the efficacy of footwear designed to mimic barefoot running. Finally, although further investigation is necessary it appears in this case that conventional shod running is superior to both barefoot running and shoes designed to mimic barefoot running, in terms of protection from running injuries. Future research should focus on prospective epidemiological analyses and the influence of different conditions footwear on the aetiology of running injuries. 
1. Cappozzo A, Catani F, Leardini A, Benedeti MG and Della CU (1995). Position and orientation in space of bones during movement: Anatomical frame definition and determination. Clinical Biomechanics, 10, p 171-178. cluster design criteria for 3-D bone movement reconstruction, IEEE Transactions on Biomedical Engineering, 44, p 1165-1174. 
3. De Koning J and Nigg B (1993). Kinematic factors affecting initial peak vertical ground reaction forces in running. In XIVth Congress of the International Symposium of Biomechanics France.

4. De Clerq D, Aerts P and Kunnon M (1994). The mechanical characteristics of the heel pad during foot strike in running: An in vivo cineradiographic study. Journal of Biomechanics, 27, p 1213-1222.

5. De wit B, De Clerq D and Aerts P (2000). Biomechanical analysis of the stance phase during barefoot and shod running. Journal of Biomechanics, 33, p 269-279.

6. Dickinson, J.A., Cook, S.D., Leinhardt, T.M. (1985). The measurement of shock waves following heel strike while running. Journal of Biomechanics, 18, p 415-22.

7. Edington CJ, Frederick EC and Cavanagh PR (1990). Rearfoot motion in distance running. In: Cavanagh P.R ed Bimechanics of distance running. Human Kinetics, Champaign, IL.

8. Erdfelder, E., Faul, F., Buchner, A., (1996). G*Power: A general power analysis program. Behaviour Research Methods Instruments and Computers, 28, p 1-11.

9. Griffin JR, Mercer JA and Dufek JS (2007). Kinematic comparison of running barefoot and in the barefoot shoe Free 5.0. Medicine and Science in Sports and Exercise, 39, S73.

10. Hardin EC, Van Den Bogert AJ and Hamill J (2004). Kinematic adaptations during running: Effects of footwear, surface and duration. Medicine and Science in Sports and Exercise, 36, p 838-844.

11. Hartveld A and Chockalingam N (2003) Shock absorption during jumping barefoot and in shoes. International Journal of Podiatric Biomechanics, 1, p16-20.

12. Lafortune, M. A., Hennig, E. M. 1991. Contribution of angular motion and gravity to tibial acceleration. Medicine and Science in Sports and Exercise, 23, p360-363. 
13. Lafortune, M. A., Hennig, E. M. 1992. Cushioning properties of footwear during walking: accelerometer and force platform measurements, Clinical Biomechanics, 7, p181-184.

14. Lieberman DE, Venkadesan M, Werbel WA, Daoud AI, D'Andrea S, Davis IS, Mang'eni RO, Pitsiladis Y. (2010) Foot strike patterns and collision forces in habitually barefoot versus shod runners. Nature, 463, p531-535.

15. Misevich KW and Cavanagh R (1984). Material aspects of modelling shoe/foot interaction. In: Frederick, E.C. Editor. Sport Shoes and Playing Surfaces. Human Kinetics Publishers, Champaign, IL, p 1-23.

16. Nigg B (2009). Biomechanical considerations on barefoot movement and barefoot shoe concepts. Footwear Science, 1, 73-79.

17. Robbins SE and Gouw GJ (1991). Athletic footwear: Unsafe due to perceptual illusions. Medicine and Science in Sports and Exercise, 22, p 217-224.

18. Robbins SE, Gouw GJ and Hanna AM (1989). Running related injury prevention through innate impact moderating behaviour, Medicine and Science in Sports and Exercise, 21, 130-139.

19. Robbins and Hanna (1987). Running Related Injury Prevention Through barefoot Adaptations, Medicine and Science in Sports and Exercise, 19, p148-156.

20. Shorten MA (2000). Running shoe design: protection and performance pp 159-169 in Marathon Medicine (Ed. D. Tunstall Pedoe) London, Royal Society of Medicine.

21. Sinclair, J., Bottoms, L., Taylor, K. \& Greenhalgh, A (2010). Tibial shock measured during the fencing lunge: the influence of footwear. Sports Biomechanics, 9, 65-71.

22. Smith L, Clarke T, Hamill C and Santopierto F (1986). The effect of soft and semi-rigid orthoses upon rearfoot movement in running. Podiatric sports medicine, 76, p 227-233. 
23. Squadrone., R, Gallozzi., C (2009). Biomechanical and physiological comparison of barefoot and two shod conditions in experienced barefoot runners. Journal of Sports Medicine and Physical Fitness, 49, p 6-13.

24. Stacoff, A., Kaelin, X., Stussi, E (1991). The effects of shoes on the torsion and rearfoot motion in running. Medicine and Science in Sports and Exercise, 23, p 482-490.

25. Warburton M (2001). Training and performance - barefoot running. Sport science, 5.

26. Willy RW and Davis IMS (2009). Kinematic and Kinetic Comparison of Running in a Neutral Cushioned Shoe and a Minimal Shoe, Medicine and Science in Sports and Exercise, 41, p 390-391. 